\title{
Antibacterial Envelope to Prevent Cardiac Implantable Device Infection
}

Tarakji, Khaldoun G ; Mittal, Suneet ; Kennergren, Charles ; Corey, Ralph ; Poole, Jeanne E ; Schloss, Edward ; Gallastegui, Jose ; Pickett, Robert A ; Evonich, Rudolph ; Philippon, François ; McComb, Janet M ; Roark, Steven F ; Sorrentino, Denise ; Sholevar, Darius ; Cronin, Edmond ; Berman, Brett ; Riggio, David ; Biffi, Mauro ; Khan, Hafiza ; Silver, Marc T ; Collier, Jack ; Eldadah, Zayd ; Wright,

David J ; Lande, Jeff D ; Lexcen, Daniel R ; Cheng, Alan ; Wilkoff, Bruce L ; WRAP-IT Investigators

\begin{abstract}
BACKGROUND Infections after placement of cardiac implantable electronic devices (CIEDs) are associated with substantial morbidity and mortality. There is limited evidence on prophylactic strategies, other than the use of preoperative antibiotics, to prevent such infections. METHODS We conducted a randomized, controlled clinical trial to assess the safety and efficacy of an absorbable, antibiotic-eluting envelope in reducing the incidence of infection associated with CIED implantations. Patients who were undergoing a CIED pocket revision, generator replacement, or system upgrade or an initial implantation of a cardiac resynchronization therapy defibrillator were randomly assigned, in a 1:1 ratio, to receive the envelope or not. Standard-of-care strategies to prevent infection were used in all patients. The primary end point was infection resulting in system extraction or revision, long-term antibiotic therapy with infection recurrence, or death, within 12 months after the CIED implantation procedure. The secondary end point for safety was procedure-related or system-related complications within 12 months. RESULTS A total of 6983 patients underwent randomization: 3495 to the envelope group and 3488 to the control group. The primary end point occurred in 25 patients in the envelope group and 42 patients in the control group (12-month Kaplan-Meier estimated event rate, $0.7 \%$ and $1.2 \%$, respectively; hazard ratio, $0.60 ; 95 \%$ confidence interval [CI], 0.36 to $0.98 ; \mathrm{P}=0.04$ ). The safety end point occurred in 201 patients in the envelope group and 236 patients in the control group (12-month Kaplan-Meier estimated event rate, $6.0 \%$ and $6.9 \%$, respectively; hazard ratio, $0.87 ; 95 \% \mathrm{CI}, 0.72$ to 1.06 ; $\mathrm{P}<0.001$ for noninferiority). The mean $( \pm \mathrm{SD})$ duration of follow-up was $20.7 \pm 8.5$ months. Major CIED-related infections through the entire follow-up period occurred in 32 patients in the envelope group and 51 patients in the control group (hazard ratio, 0.63; 95\% CI, 0.40 to 0.98). CONCLUSIONS Adjunctive use of an antibacterial envelope resulted in a significantly lower incidence of major CIED infections than standard-of-care infection-prevention strategies alone, without a higher incidence of complications. (Funded by Medtronic; WRAP-IT ClinicalTrials.gov number, NCT02277990.).
\end{abstract}

DOI: https://doi.org/10.1056/NEJMoa1901111

Posted at the Zurich Open Repository and Archive, University of Zurich

ZORA URL: https://doi.org/10.5167/uzh-173417

Journal Article

Published Version

Originally published at: 
Tarakji, Khaldoun G; Mittal, Suneet; Kennergren, Charles; Corey, Ralph; Poole, Jeanne E; Schloss, Edward; Gallastegui, Jose; Pickett, Robert A; Evonich, Rudolph; Philippon, François; McComb, Janet M; Roark, Steven F; Sorrentino, Denise; Sholevar, Darius; Cronin, Edmond; Berman, Brett; Riggio, David; Biffi, Mauro; Khan, Hafiza; Silver, Marc T; Collier, Jack; Eldadah, Zayd; Wright, David J; Lande, Jeff D; Lexcen, Daniel R; Cheng, Alan; Wilkoff, Bruce L; WRAP-IT Investigators (2019). Antibacterial Envelope to Prevent Cardiac Implantable Device Infection. New England Journal of Medicine, 380(20):1895-1905. DOI: https://doi.org/10.1056/NEJMoa1901111 


\title{
The NEW ENGLAN D JOURNAL of MEDICINE
}

\section{Antibacterial Envelope to Prevent Cardiac Implantable Device Infection}

\author{
Khaldoun G. Tarakji, M.D., M.P.H., Suneet Mittal, M.D., Charles Kennergren, M.D., Ph.D., Ralph Corey, M.D., \\ Jeanne E. Poole, M.D., Edward Schloss, M.D., Jose Gallastegui, M.D., Robert A. Pickett, M.D., \\ Rudolph Evonich, M.D., François Philippon, M.D., Janet M. McComb, M.D., Steven F. Roark, M.D., \\ Denise Sorrentino, M.D., Darius Sholevar, M.D., Edmond Cronin, M.B., B.Ch., B.A.O., Brett Berman, M.D., \\ David Riggio, M.D., Mauro Biffi, M.D., Hafiza Khan, M.D., Marc T. Silver, M.D., Jack Collier, M.D., \\ Zayd Eldadah, M.D., Ph.D., David J. Wright, M.D., Jeff D. Lande, Ph.D., Daniel R. Lexcen, Ph.D., \\ Alan Cheng, M.D., and Bruce L. Wilkoff, M.D., for the WRAP-IT Investigators*
}

ABSTRACT

\section{BACKGROUND}

Infections after placement of cardiac implantable electronic devices (CIEDs) are associated with substantial morbidity and mortality. There is limited evidence on prophylactic strategies, other than the use of preoperative antibiotics, to prevent such infections.

METHODS

We conducted a randomized, controlled clinical trial to assess the safety and efficacy of an absorbable, antibiotic-eluting envelope in reducing the incidence of infection associated with CIED implantations. Patients who were undergoing a CIED pocket revision, generator replacement, or system upgrade or an initial implantation of a cardiac resynchronization therapy defibrillator were randomly assigned, in a 1:1 ratio, to receive the envelope or not. Standard-of-care strategies to prevent infection were used in all patients. The primary end point was infection resulting in system extraction or revision, long-term antibiotic therapy with infection recurrence, or death, within 12 months after the CIED implantation procedure. The secondary end point for safety was procedure-related or system-related complications within 12 months.

RESULTS

A total of 6983 patients underwent randomization: 3495 to the envelope group and 3488 to the control group. The primary end point occurred in 25 patients in the envelope group and 42 patients in the control group (12-month Kaplan-Meier estimated event rate, 0.7\% and $1.2 \%$, respectively; hazard ratio, $0.60 ; 95 \%$ confidence interval [CI], 0.36 to 0.98 ; $\mathrm{P}=0.04)$. The safety end point occurred in 201 patients in the envelope group and 236 patients in the control group (12-month Kaplan-Meier estimated event rate, $6.0 \%$ and $6.9 \%$, respectively; hazard ratio, $0.87 ; 95 \% \mathrm{CI}, 0.72$ to $1.06 ; \mathrm{P}<0.001$ for noninferiority). The mean $( \pm S D)$ duration of follow-up was $20.7 \pm 8.5$ months. Major CIED-related infections through the entire follow-up period occurred in 32 patients in the envelope group and 51 patients in the control group (hazard ratio, $0.63 ; 95 \% \mathrm{CI}, 0.40$ to 0.98 ).

CONCLUSIONS

Adjunctive use of an antibacterial envelope resulted in a significantly lower incidence of major CIED infections than standard-of-care infection-prevention strategies alone, without a higher incidence of complications. (Funded by Medtronic; WRAP-IT ClinicalTrials.gov number, NCT02277990.)
The authors' affiliations are listed in the Appendix. Address reprint requests to Dr. Tarakji at the Cleveland Clinic, 9500 Euclid Ave., J2-2, Cleveland, OH 44195, or at tarakjk@ccf.org.

*A full list of the WRAP-IT investigators is provided in the Supplementary Appendix, available at NEJM.org.

This article was published on March 17, 2019, at NEJM.org.

N Engl J Med 2019;380:1895-905. DOI: 10.1056/NEJMoa1901111

Copyright (c) 2019 Massachusetts Medical Society. 
A Quick Take is available at NEJM.org
T IS ESTIMATED THAT 1.5 MILLION PATIENTS receive cardiac implantable electronic devices (CIEDs) worldwide every year. ${ }^{1}$ Despite the use of various antibiotic prophylaxis regimens and advocacy of best surgical practices by society consensus statements, ${ }^{2,3}$ infections after placement of CIEDs remain a major complication and are associated with substantial morbidity, mortality, and health care utilization. ${ }^{4-8}$ There is limited evidence on prophylactic strategies, other than the use of preoperative antibiotics, to reduce infection. Although a number of studies support the use of local antibiotic delivery, ${ }^{9-14}$ a large, multicenter, randomized, controlled trial has been lacking. An absorbable, multifilament mesh envelope (TYRX Absorbable Antibacterial Envelope, Medtronic) is available to improve CIED stabilization in the subcutaneous pocket and to elute the antibiotics minocycline and rifampin. The aim of this randomized clinical trial was to evaluate the safety and effectiveness of the envelope in reducing CIED infection as adjunctive therapy to standard infection-prevention strategies.

METHODS

TRIAL DESIGN

The Worldwide Randomized Antibiotic Envelope Infection Prevention Trial (WRAP-IT) was a multicenter, randomized, controlled, prospective, singleblind, postmarketing, interventional clinical trial. It compared the incidence of major CIED infections through 12 months after implantation among patients who received an absorbable antibacterial envelope with the incidence among patients who did not receive the envelope.

Standard-of-care infection-prevention strategies (preprocedure intravenous antibiotics and sterile technique) were used in all patients. Patients were randomly assigned in a 1:1 ratio, with stratification according to trial site and device type (pacemaker or cardiac resynchronization therapy pacemaker [CRT-P] vs. implantable cardioverterdefibrillator [ICD] or cardiac resynchronization therapy defibrillator [CRT-D]), to receive the envelope during their CIED procedure (envelope group) or not to receive the envelope (control group). (An image of the envelope is provided in Fig. S1 in the Supplementary Appendix, available with the full text of this article at NEJM.org.)
Randomly assigned patients were followed at hospital discharge and at 6-month intervals until all the patients completed a minimum of 12 months of follow-up. Patients were unaware of the trial-group assignments during the followup period. Adverse events, including signs and symptoms of CIED infection, were assessed at each follow-up visit. The trial design has been described previously. ${ }^{15}$

\section{TRIAL OVERSIGHT}

The steering committee (Table S1 in the Supplementary Appendix) designed and oversaw the conduct of the trial and data analyses in collaboration with the sponsor, Medtronic. The protocol, approved by the ethics committee at each participating institution and associated national and local regulatory agencies, and statistical analysis plan are available at NEJM.org. All the patients provided written informed consent.

A clinical-events committee (Table S2 in the Supplementary Appendix), whose members were unaware of the randomization assignments, adjudicated all deaths and adverse events potentially related to the implanted system or procedure, including CIED infections. An independent data and safety monitoring committee (Table S3 in the Supplementary Appendix), managed by the Cleveland Clinic Coordinating Center for Clinical Research, met periodically during trial enrollment and reviewed the formal interim analysis, which occurred when 5165 patients were enrolled and 3189 6-month postprocedure visits had been accrued, as prespecified by the statistical analysis plan. The first draft of the manuscript was prepared by the steering committee, whose members had unrestricted access to the data, and was reviewed and edited by all the authors. All the authors vouch for the accuracy and completeness of the data and analyses reported and for the fidelity of the trial to the protocol.

\section{TRIAL DEVICE}

The TYRX envelope is an absorbable single-use envelope designed to hold a CIED when the device is implanted in the body. The envelope is constructed from a multifilament knitted mesh and coated with an absorbable polymer mixed with minocycline and rifampin, which elutes the antibiotics into the local tissue for a minimum 
of 7 days. The envelope is fully absorbed in approximately 9 weeks.

\section{PATIENT POPULATION}

As described previously, ${ }^{15}$ our enrollment strategy was to include patients who were perceived to be at increased risk for CIED infection on the basis of historical studies in the literature. This population included patients undergoing CIED generator replacement or a system upgrade with or without new leads, those undergoing CIED pocket or lead revision, and those undergoing an initial CRT-D procedure. ${ }^{13,16}$ Detailed inclusion and exclusion criteria are provided in Table S4 in the Supplementary Appendix. According to the protocol, pacemaker (including CRT-P) randomizations were capped at $25 \%$ of the target sample size.

\section{END POINTS}

The trial had one primary end point and three secondary end points. The primary end point was major CIED infection within 12 months (365 days) after the CIED procedure. CIED infection was defined as superficial cellulitis in the region of the CIED pocket with wound dehiscence, erosion, or purulent drainage; deep incisional or space (pocket) surgical-site infection that met the Centers for Disease Control and Prevention criteria, independent of time from surgery; persistent bacteremia; or endocarditis. Major CIED infections were defined as infections that resulted in CIED system removal, an invasive CIED procedure (e.g., pocket revision without removal), treatment with long-term antibiotic therapy (if the patient was not a candidate for system removal) with infection recurrence after discontinuation of antibiotic therapy, or death. CIED infections that did not meet one or more of the criteria for major infection were classified as minor CIED infections. Secondary end points were CIED procedure-related or system-related complications within 12 months after the procedure, major or minor CIED infection within 12 months, and major CIED infection regardless of when it occurred. Death from any cause was an ancillary end point of interest.

\section{STATISTICAL ANALYSIS}

The statistical methods and sample-size justification have been described previously. ${ }^{15}$ Briefly, we calculated that a sample size of 6988 ran- domly assigned patients would provide $90 \%$ power with the use of a Cox proportional-hazards model to evaluate the primary end point. Samplesize calculation was performed with the use of simulation under the following assumptions: a rate of major CIED infection at 12 months after the procedure of $2 \%$ in the control group $(2.4 \%$ among patients receiving an ICD or CRT-D and $0.6 \%$ among those receiving a pacemaker or CRT-P), a $50 \%$ lower incidence of major CIED infection in the envelope group than in the control group, implantation of an ICD or a CRT-D in $75 \%$ of the patients, an annualized attrition rate of up to $15 \%$, and one interim analysis to assess success and futility with the use of group-sequential stopping boundaries to maintain an overall type I error rate of $5 \%$.

The interim analysis occurred when 3189 randomly assigned patients completed their 6-month visit and stopping boundaries were not met. The primary end point was evaluated with the use of a Cox proportional-hazards regression model stratified according to device type (pacemaker or CRT-P vs. ICD or CRT-D). Kaplan-Meier methods were used to construct event-rate plots. Time to event was set to the interval from the time of the procedure (or randomization) to the time of the first major CIED infection within 365 days. Data on patients who did not meet the primary end point were censored at their last follow-up visit or day 365 , whichever occurred first. Homogeneity of the treatment effect was tested for subgroups by the inclusion of an interaction term for subgroup according to treatment.

Similar stratified Cox models were also used to compare the two trial groups with respect to the secondary end points and death, with the maximum follow-up time set to 365 days or the maximum observed days with respect to the endpoint definition. In addition, CIED procedurerelated or system-related complications were compared between the groups with the use of a noninferiority margin for the hazard ratio of 1.33 (i.e., the hazard ratio for complications in the envelope group vs. the control group must be significantly lower than 1.33). All end points were evaluated on an intention-to-treat basis with the exception of the secondary end point of CIED complications, which was evaluated on an as-treated basis because a noninferiority hypothesis was tested. ${ }^{17}$ The Holm multiple-testing pro- 


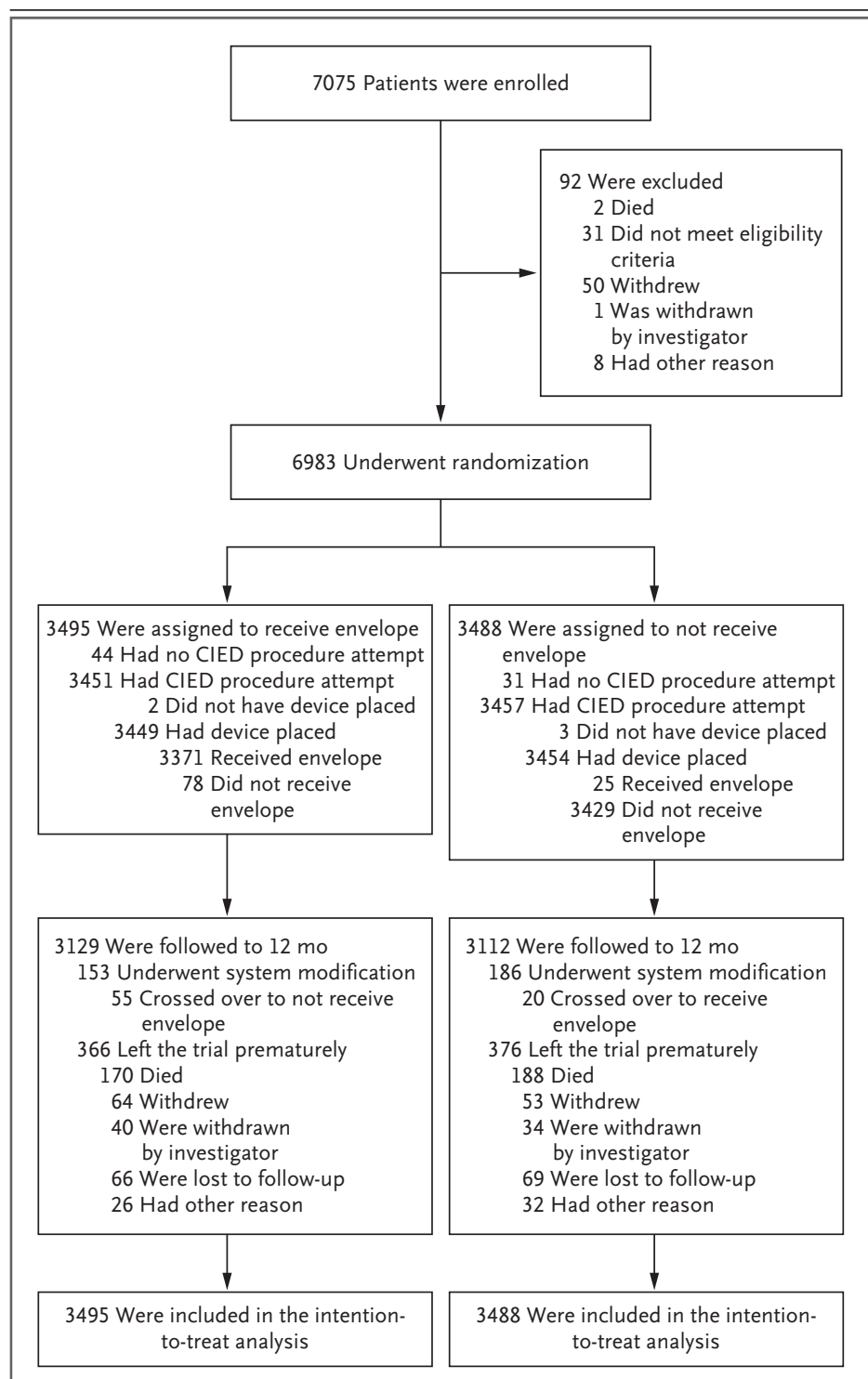

Figure 1. Enrollment, Randomization, and Follow-up of the Trial Patients. CIED denotes cardiac implantable electronic device. cedure was used to control the type I error for the three secondary objective analyses.

The sample-size calculation and all analyses were performed with the use of the R statistical package (R Project for Statistical Computing) or SAS software, version 9.4 (SAS Institute). P values of less than 0.0488 for the primary and secondary end points were considered to indicate statistical significance (with adjustment for the interim analysis). Independent statistical validation of the trial results was conducted by the Cleveland Clinic Coordinating Center for Clinical Research.
RESULTS

\section{PATIENTS}

Patient recruitment occurred from January 2015 through July 2017, with 7075 patients enrolled and 776 implanting physicians at 181 centers in 25 countries within North America, Europe, Asia, and South America (see the Supplementary Appendix); 92 patients did not undergo randomization (Fig. 1). Among the remaining 6983 patients, 3495 were assigned to the envelope group and 3488 to the control group. Patient characteristics at baseline were balanced between the two groups except for a higher percentage of use of immunosuppressive agents in the control group (Table 1). The mean $( \pm S D)$ age was $70.1 \pm 12.5$ years; $28.3 \%$ of the patients were women.

\section{PROCEDURE CHARACTERISTICS}

Procedure characteristics were balanced between the two groups. A total of 3429 patients who were assigned to the control group did not receive the envelope, and 3371 patients who were assigned to the envelope group received the envelope at the time of the CIED procedure. In the envelope group, the envelope was not successfully implanted in 10 procedures owing to limited pocket space; thus, the envelope was successfully implanted in $99.7 \%$ of procedure attempts (3371 of 3381 attempts) by 646 implanting physicians. The mean procedure time did not differ significantly between the two groups $(56.3 \pm 46.2$ minutes in the envelope group and $55.0 \pm 48.0$ minutes in the control group; between-group difference, 1.3 minutes; 95\% confidence interval [CI], -0.9 to 3.5 ).

\section{PATIENT FOLLOW-UP}

Patients were followed for a mean of $20.7 \pm 8.5$ months, with $89.4 \%$ of patients completing at least 12 months of follow-up (Fig. 1); the duration of follow-up did not differ significantly between the two groups. During the 12-month follow-up, 181 system revisions occurred in 153 patients in the envelope group and 229 in 186 patients in the control group (annualized rate, 0.06 and 0.07 , respectively; rate ratio, $0.79 ; 95 \%$ CI, 0.65 to 0.96 ).

\section{PRIMARY END POINT}

Within 12 months after the index CIED procedure, there were 30 major infections in 25 patients in the envelope group and 45 major infections in 


\begin{tabular}{|c|c|c|}
\hline Characteristic & Envelope ( $N=3495)$ & Control $(\mathrm{N}=3488)$ \\
\hline \multicolumn{3}{|l|}{ Age } \\
\hline No. of patients evaluated & 3490 & 3485 \\
\hline Mean $-\mathrm{yr}$ & $70.0 \pm 12.6$ & $70.1 \pm 12.4$ \\
\hline Female sex — no. (\%) & $997(28.6)$ & $976(28.0)$ \\
\hline \multicolumn{3}{|l|}{ Body-mass indext' } \\
\hline No. of patients evaluated & 3477 & 3468 \\
\hline Mean & $29.1 \pm 6.1$ & $29.2 \pm 6.3$ \\
\hline \multicolumn{3}{|l|}{ Medical history - no./total no. (\%) } \\
\hline Cardiomyopathy & $2364 / 3489(67.8)$ & $2374 / 3483(68.2)$ \\
\hline Coronary artery disease & $1449 / 3489(41.5)$ & $1478 / 3483(42.4)$ \\
\hline Myocardial infarction & $982 / 3489(28.1)$ & $934 / 3483(26.8)$ \\
\hline COPD & $455 / 3489(13.0)$ & $418 / 3483(12.0)$ \\
\hline Diabetes & $1080 / 3489(31.0)$ & $1085 / 3483(31.2)$ \\
\hline Renal dysfunction & $585 / 3489(16.8)$ & $554 / 3483(15.9)$ \\
\hline \multicolumn{3}{|l|}{ Cardiovascular surgical history — no./total no. (\%) } \\
\hline CABG & $731 / 3489(21.0)$ & $760 / 3483(21.8)$ \\
\hline Valve surgery & $323 / 3489(9.3)$ & $303 / 3483(8.7)$ \\
\hline \multicolumn{3}{|l|}{ No. of previous CIEDs } \\
\hline No. of patients evaluated & 3487 & 3482 \\
\hline Mean & $1.2 \pm 0.9$ & $1.2 \pm 1.0$ \\
\hline \multicolumn{3}{|l|}{ Time since first CIED } \\
\hline No. of patients evaluated & 2810 & 2747 \\
\hline Mean $-\mathrm{yr}$ & $9.0 \pm 4.9$ & $9.1 \pm 5.1$ \\
\hline Previous CIED infection — no./total no. (\%) & $45 / 3489(1.3)$ & $53 / 3483(1.5)$ \\
\hline \multicolumn{3}{|l|}{ Baseline medications - no./total no. (\%) } \\
\hline Antiplatelet & $2007 / 3490(57.5)$ & $1972 / 3485(56.6)$ \\
\hline Anticoagulant & $1377 / 3490(39.5)$ & $1390 / 3485$ (39.9) \\
\hline Antibiotic & $36 / 3490(1.0)$ & $37 / 3485(1.1)$ \\
\hline Immunosuppressive & $48 / 3490(1.4)$ & $85 / 3485(2.4)$ \\
\hline Insulin & $348 / 3490(10.0)$ & $375 / 3485(10.8)$ \\
\hline Oral antidiabetic & $615 / 3490(17.6)$ & $620 / 3485(17.8)$ \\
\hline \multicolumn{3}{|l|}{ Infection-management strategy — no./total no. (\%)』 } \\
\hline Periprocedure antibiotic & $3402 / 3450(98.6)$ & $3413 / 3457(98.7)$ \\
\hline Preprocedure antibiotic & $3250 / 3450(94.2)$ & $3291 / 3457(95.2)$ \\
\hline Antibiotic during procedure & $690 / 3450(20.0)$ & $685 / 3457(19.8)$ \\
\hline Postprocedure antibiotic & $987 / 3450(28.6)$ & $1058 / 3457(30.6)$ \\
\hline Pocket wash & $2539 / 3450(73.6)$ & $2610 / 3457(75.5)$ \\
\hline \multicolumn{3}{|l|}{$\mathrm{CIED}$ type planned at randomization - no. (\%) } \\
\hline \multicolumn{3}{|l|}{ Low power } \\
\hline Pacemaker & $723(20.7)$ & $709(20.3)$ \\
\hline CRT-P & $133(3.8)$ & $157(4.5)$ \\
\hline \multicolumn{3}{|l|}{ High power } \\
\hline$I C D$ & $964(27.6)$ & $909(26.1)$ \\
\hline CRT-D & $1675(47.9)$ & $1713(49.1)$ \\
\hline Procedure attempted but no CIED implanted - no. (\%) & $2(0.1)$ & $3(0.1)$ \\
\hline No procedure attempted — no. (\%) & $44(1.3)$ & $31(0.9)$ \\
\hline
\end{tabular}

* Plus-minus values are means \pm SD. There were no significant differences between the two groups except for the use of immunosuppressive agents $(P=0.001)$, with values not adjusted for multiple testing; the standardized difference for immunosuppressive agents does not suggest imbalance, since the absolute value does not exceed 0.1. CABG denotes coronary-artery bypass grafting, CIED cardiac implantable electronic device, COPD chronic obstructive pulmonary disease, CRT-D cardiac resynchronization therapy defibrillator, and CRT-P cardiac resynchronization therapy pacemaker.

$\dagger$ The body-mass index is the weight in kilograms divided by the square of the height in meters.

Shown are patients with a CIED infection more than 12 months before trial enrollment.

$\int$ Counts and percentages indicate patients with procedure attempts.

The New England Journal of Medicine 
42 patients in the control group (12-month KaplanMeier estimated event rate, $0.7 \%$ and $1.2 \%$, respectively; hazard ratio, $0.60 ; 95 \% \mathrm{CI}, 0.36$ to $0.98 ; \mathrm{P}=0.04$ ) (Fig. $2 \mathrm{~A}$ and Table 2). With respect to the first major infection in each patient, 17 were endocarditis or bacteremia and 50 were pocket infections. There were fewer pocket infections but numerically more endocarditis or bacteremia infections in the envelope group than in the control group (Table 2, and Fig. S2 in the Supplementary Appendix). The most common major CIED criterion met in both groups was system removal (Table S6 in the Supplementary Appendix). Of the 36 organisms identified in the initial infection events, 23 were staphylococcus bacteria (Tables S7 and S8 in the Supplementary Appendix). Data on antibiotic susceptibility were not collected.

\section{SECONDARY END POINTS AND DEATH}

We implemented the Holm procedure to control for multiple comparisons for the secondary end points. As shown in Table S9 in the Supplementary Appendix, the secondary end point that is listed first (complications through 12 months) passed the Holm criteria, but the subsequent two end points that are listed did not.

Complications that occurred within 12 months after the index CIED procedure and that were related to the CIED system or procedure occurred in 201 patients in the envelope group and 236 patients in the control group (12-month Kaplan-Meier estimated event rate, 6.0\% and $6.9 \%$, respectively; hazard ratio, $0.87 ; 95 \% \mathrm{CI}$, 0.72 to 1.06 ; $\mathrm{P}<0.001$ for noninferiority) (Fig. S3 in the Supplementary Appendix). Excluding the primary end point of major infections, the KaplanMeier 12-month complication event rates were $5.7 \%$ in the envelope group and $5.9 \%$ in the control group.

Major or minor CIED infections within 12 months occurred in 50 patients in the envelope group and 75 patients in the control group (12-month Kaplan-Meier estimated event rate, $1.5 \%$ and $2.2 \%$, respectively; hazard ratio, 0.67 ; $95 \%$ CI, 0.47 to 0.96 ). Through the entire followup period, major CIED infections occurred in 32 patients in the envelope group and 51 patients in the control group (36-month Kaplan-Meier estimated event rate, $1.3 \%$ and $1.9 \%$, respectively; hazard ratio, 0.63 ; $95 \%$ CI, 0.40 to 0.98 ) (Fig. 2B).

A total of 349 deaths occurred in the envelope group and 365 deaths occurred in the control group throughout the follow-up period (36-month Kaplan-Meier rate of death, $17.4 \%$ and $17.8 \%$, respectively; hazard ratio, 0.96 ; $95 \% \mathrm{CI}, 0.83$ to 1.11) (Table $S 9$ in the Supplementary Appendix).

\section{SUBGROUP ANALYSIS}

A subgroup analysis was conducted to test for interaction among various baseline variables for the primary end point through 12 months. None of the differences between subgroups, inclusive of age, sex, or baseline characteristics, were significant. In the subgroup receiving a high-power device (ICD or CRT-D), major CIED infections occurred in 18 patients in the envelope group and 35 patients in the control group (12-month Kaplan-Meier estimated event rate, $0.7 \%$ and $1.4 \%$, respectively; hazard ratio, $0.51 ; 95 \% \mathrm{CI}$, 0.29 to 0.90 ). In the subgroup receiving a lowpower device (pacemaker or CRT-P), major CIED infections occurred in 7 patients in the envelope group and 7 patients in the control group (12-month Kaplan-Meier estimated event rate, $0.9 \%$ and $0.8 \%$, respectively; hazard ratio, 1.02 ; $95 \%$ CI, 0.36 to 2.92). The interaction effect for subgroup according to device type was not significant (Fig. 3).

In a post hoc analysis involving the patients who received an initial CRT-D, 536 were assigned to the envelope group and 586 to the control group. Major CIED infections occurred in 7 patients in the envelope group and 3 patients in the control group (12-month Kaplan-Meier estimated event rate, $1.3 \%$ and $0.5 \%$, respectively; hazard ratio, 2.55 ; $95 \%$ CI, 0.66 to 9.85 ).

\section{DISCUSSION}

WRAP-IT was a randomized, controlled clinical trial to assess the safety and efficacy of the TYRX absorbable antibiotic-eluting envelope. In a population of patients who were at increased risk for CIED pocket infection, the envelope was significantly more effective at preventing infection than standard infection-control strategies alone. The efficacy objective was met, with $40 \%$ fewer patients in the envelope group than in the control group having a major infection through 12 months of follow-up. The envelope was successfully implanted in $99.7 \%$ of procedure attempts, and the safety objective was met, because the envelope group did not have a higher incidence 


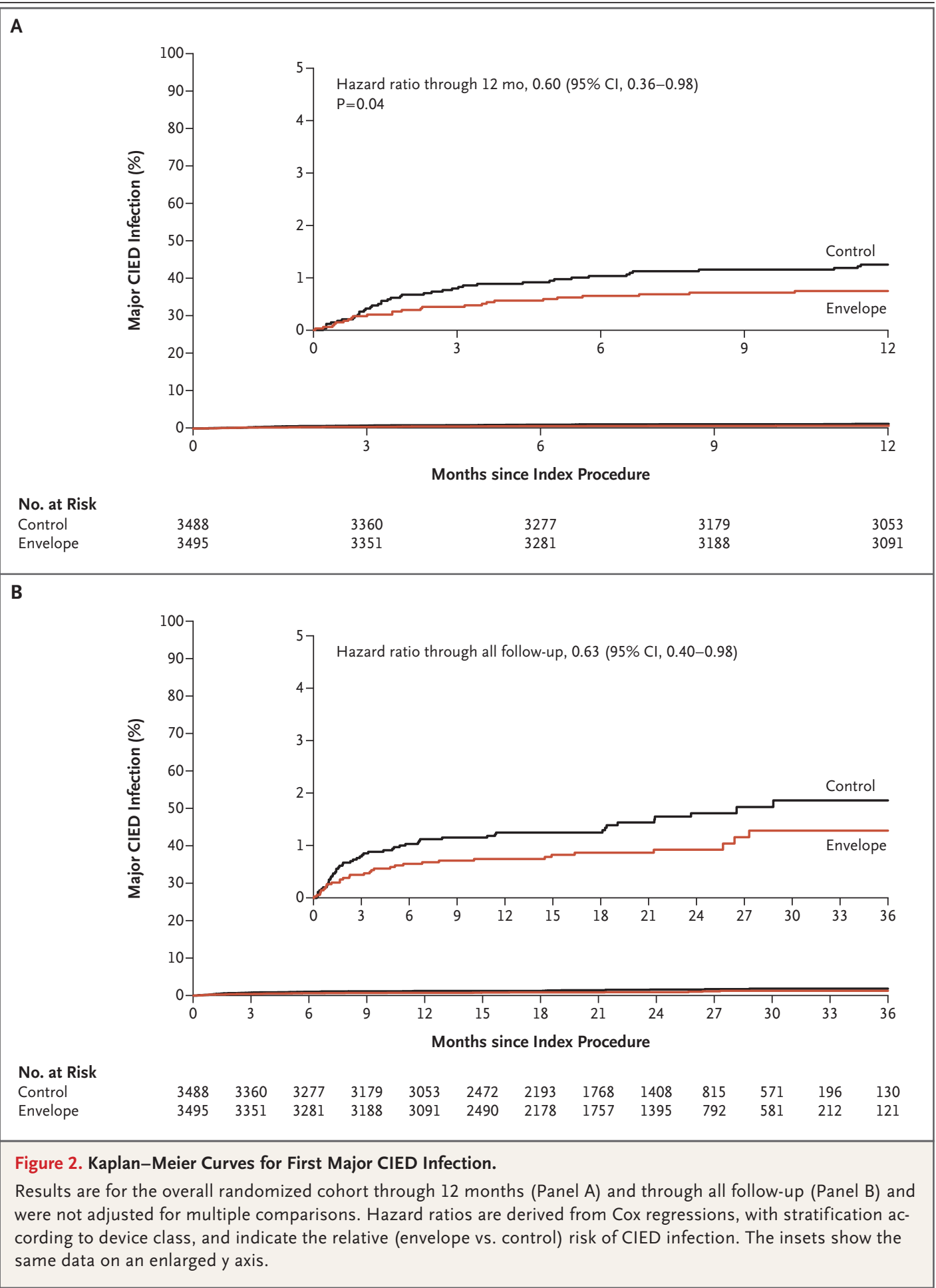

of CIED procedure-related or system-related complications than the control group.

CIED infection is a rare but serious event, and its management requires prolonged hospitaliza- tion, which involves device and lead extraction with adjunctive antibiotic therapy. ${ }^{18}$ The risk of major complications with lead extraction is low, approximately 1 to $2 \%$, but these can be fatal. ${ }^{19,20}$ 


\begin{tabular}{|lcccc|}
\hline Table 2. Summary of Initial Major CIED Infections within 12 Months. \\
\hline & $\begin{array}{c}\text { Envelope } \\
(\mathbf{N}=3495)\end{array}$ & $\begin{array}{c}\text { Control } \\
\mathbf{( N = 3 4 8 8 )}\end{array}$ & $\begin{array}{c}\text { Total } \\
(\mathbf{N}=6983)\end{array}$ & $\begin{array}{c}\text { Hazard Ratio } \\
\mathbf{( 9 5 \% ~ C I )}\end{array}$ \\
$\begin{array}{l}\text { End Point } \\
\begin{array}{l}\text { Primary end point: major CIED } \\
\text { infection within 12 mo }\end{array}\end{array}$ & $25(0.7)$ & $42(1.2)$ & $67(1.0)$ & $0.60(0.36-0.98) *$ \\
$\begin{array}{l}\text { Type of major CIED infection } \\
\text { Pocket infection }\end{array}$ & $14(0.4)$ & $36(1.0)$ & $50(0.7)$ & $0.39(0.21-0.72)$ \\
Bacteremia or endocarditis & $11(0.3)$ & $6(0.2)$ & $17(0.2)$ & $1.57(0.61-4.05)$ \\
\hline
\end{tabular}

$* \mathrm{P}=0.04$

Despite proper management of CIED infection, morbidity and both short- and long-term mortality remain high. ${ }^{4-7}$ One randomized trial has shown a reduction in the risk of infection related to CIED procedures. ${ }^{21,22}$ In a trial of 1000 consecutive CIED procedures at a single center, intravenous administration of cefazolin before the procedure reduced the risk of infection by $81 \%{ }^{21}$ More recently, an unblinded, cluster-randomized, crossover trial (Prevention of Arrhythmia Device Infection Trial [PADIT]) was conducted to assess the effect of incremental prophylaxis antibiotics before, during, and after the CIED procedure. ${ }^{22}$ The use of the TYRX envelope was prohibited. The primary end point was not achieved because rates of hospitalization due to infection were not significantly reduced with incremental antibiotics. In our trial, $98.7 \%$ of the patients received periprocedure antibiotics, $74.5 \%$ received pocket wash, and $29.6 \%$ received postprocedure antibiotics (Table 1).

These results add to the existing body of literature on the safety and efficacy of the envelope in reducing CIED infections. ${ }^{9-13}$ Although the use of the envelope may require dissection of a slightly larger CIED pocket, we did not observe a significant difference in the rate of complications (e.g., hematoma) or procedure time that could be attributed to this. There were fewer system revisions in the envelope group than in the control group and no complications due to allergy to the envelope mesh, polymer, or antibiotics. Because the envelope is designed to prophylactically prevent deep incisional pocket infections, the trial excluded patients at high risk for systemic infection due to other sources, as well as patients with existing infection. We observed $61 \%$ fewer deep incisional pocket infections in the envelope group than in the control group (Table 2, and Fig. S2 in the Supplementary Appendix), and the effect was sustained through 3 years of follow-up. The total number of all CIED infections (both major and minor) during 12 months of follow-up was lower in the envelope group than in the control group, although the envelope is not expected to prevent superficial infections, which tend to resolve without CIED removal. Why there were numerically more cases of endocarditis or bacteremia in the envelope group is unclear.

Infections leading to an invasive procedure with or without CIED system removal, longerterm suppressive antibiotic therapy, or death occurred in $1.4 \%$ of standard-of-care patients receiving high-power devices (ICD or CRT-D) and $0.8 \%$ of standard-of-care patients receiving lowpower devices (pacemaker or CRT-P); this finding is in line with published trials. ${ }^{9,10,13}$ CIED infections occurred in $0.5 \%$ of standard-of-care patients receiving an initial CRT-D, which is lower than in published trials. ${ }^{13,23}$ The beneficial effects of the envelope in preventing major CIED infection in 12 months were more pronounced in patients with high-power devices than in those with low-power devices or an initial CRT-D. In general, patients with low-power devices and those who received an initial CRT-D were observed to have low infection rates, which limits the assessment of infection reduction with the envelope. It is notable that of patients in the control group, four patients with low-power devices and two patients with an initial CRT-D had a pocket infection within 12 months. Subgroup analyses of the primary end point according to age, sex, and baseline characteristics also showed no significant differences in effect, but these 


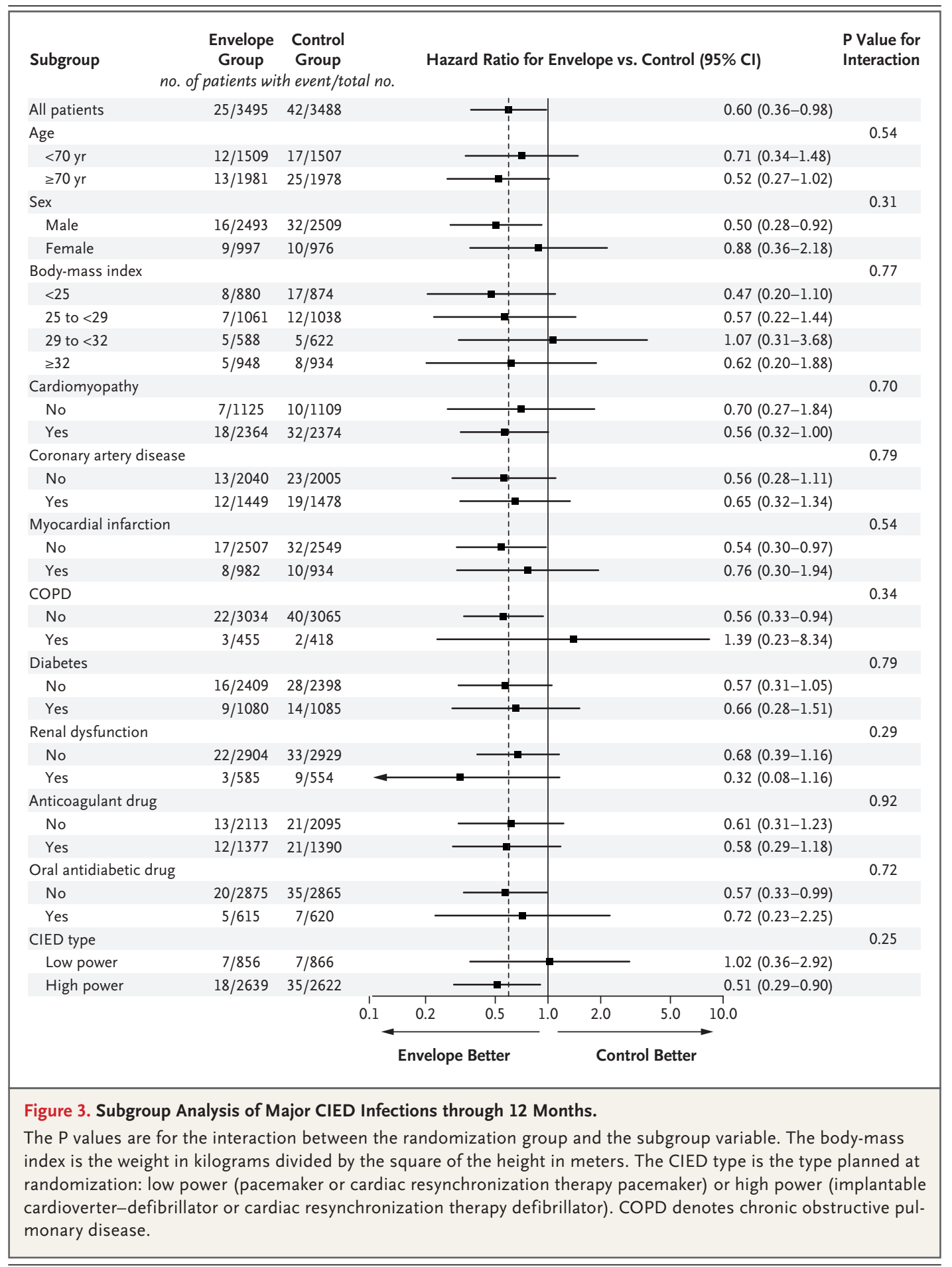

results should be interpreted with caution owing to the low event rates.

Some limitations should be considered when interpreting the results of this trial. First, consecutive patients were not enrolled because this trial was limited to patients receiving generators from one device manufacturer (leads from other manufacturers were permitted). Second, the envelope was commercially available at the time of the trial, which may have influenced participa- 
tion in the trial. Third, the use of immunosuppressive agents was not balanced between the trial groups; however, among these patients, there was only one major infection within the first 12 months, and therefore this is unlikely to influence interpretation of the results. Fourth, the lack of data on antibiotic susceptibility limits our ability to address the unknown risk of the development of antibiotic resistance. Fifth, periprocedure and postprocedure infection-prevention strategies including antibiotic use were not controlled; however, there is not yet clear evidence that a particular strategy influences the infection rate.

In conclusion, adjunctive use of an antibacterial envelope resulted in a $40 \%$ lower incidence of major CIED infection than standard-of-care infection-prevention strategies alone. Patients who received the envelope did not have more procedure-related or system-related complications than those who did not receive it.

A data sharing statement provided by the authors is available with the full text of this article at NEJM.org.

Supported by Medtronic.

Dr. Tarakji reports receiving consulting fees and lecture fees from Medtronic and consulting fees from AliveCor; Dr. Mittal, receiving consulting fees from Abbott Laboratories, Boston Scientific, and Medtronic; Dr. Corey, receiving advisory board fees and consulting fees from Arsanis, ContraFect, Melinta Therapeutics, and Paratek Pharmaceuticals, consulting fees from Basilea Pharmaceutica, Bayer, Motif Bio, Quintiles, the Medicines Company, Theravance Biopharma, Cempra, Meiji Seika Pharma, Regeneron Pharmaceuticals, and scPharmaceuticals, advisory board fees from Pfizer and Tetraphase Pharmaceuti- cals, and fees for serving on an adjudication committee from Bio2 Medical and Novella Clinical; Dr. Poole, receiving lecture fees from Medtronic, advisory board fees from Boston Scientific, grant support and consulting fees from Kestra, and fees for serving on a trial committee from EBR Systems; Dr. Schloss, receiving consulting fees, lecture fees, and travel support from Medtronic and Boston Scientific; Dr. Pickett, receiving funding and technology instruction from Medtronic and grant support from Boston Scientific; Dr. Evonich, receiving funding, paid to his institution, and nonfinancial support from Medtronic, Boehringer Ingelheim, AstraZeneca, and Eli Lilly, funding, paid to his institution, from SentreHEART, Coherex Medical, and Hamilton Health Sciences through the Population Health Research Institute, funding, paid to his institution, nonfinancial support, and travel support from Boston Scientific, and nonfinancial support from Abbott Laboratories, Allergan, Biosense Webster, CorMatrix Cardiovascular, E.R. Squibb and Sons, Janssen Pharmaceuticals, Pfizer, Sanofi-Aventis, Terumo Medical, Zoll Services, CVRx, Ekos, Novartis Pharmaceuticals, Preventice Services, Roxwood Medical, and Siemens Medical Solutions USA; Dr. Philippon, receiving lecture fees and serving as a proctor for Medtronic; Dr. Biffi, receiving fees for an educational activity and fees for serving on a speakers bureau from Boston Scientific, Biotronik, and Medtronic; Dr. Khan, receiving fees for serving on a speakers bureau from Sanofi, Preventice Services, Bristol-Myers Squibb/Pfizer, and Janssen and grant support, paid to Baylor Research Institute, from Medtronic; Dr. Silver, receiving fees for serving on a speakers bureau from Medtronic; Dr. Wright, receiving advisory board fees from Medtronic and grant support from Boston Scientific; Dr. Lande, being employed by Medtronic; Dr. Lexcen, being employed by and holding stock in Medtronic; Dr. Cheng, being employed by Medtronic; and Dr. Wilkoff, receiving consulting fees from Medtronic, Philips, and Abbott Laboratories. No other potential conflict of interest relevant to this article was reported.

Disclosure forms provided by the authors are available with the full text of this article at NEJM.org.

We thank Joanne Krueger, B.A., and Sarah Willey, M.S., for their management of the trial and Swathi Seshadri, Ph.D., for assistance in the preparation of an earlier version of the manuscript.

\section{APPENDIX}

The authors' affiliations are as follows: the Cleveland Clinic, Cleveland (K.G.T., B.L.W.), and the Lindner Research Center, Cincinnati (E.S.) - both in Ohio; Valley Health System, Ridgewood (S.M.), and Lourdes Cardiology Services, Cherry Hill (D. Sholevar) — both in New Jersey; Sahlgrenska University Hospital, Göteborg, Sweden (C.K.); Duke Clinical Research Institute, Durham (R.C.), and WakeMed Heart and Vascular, WakeMed Health and Hospitals, Raleigh (M.T.S.) - both in North Carolina; University of Washington School of Medicine, Seattle (J.E.P.); Clearwater Cardiovascular and Interventional Consultants, Safety Harbor (J.G.), and Cardiology Associates of Gainesville, Gainesville (S.F.R.) — both in Florida; Saint Thomas Research Institute, Nashville (R.A.P.); Upper Michigan Cardiovascular Associates, Marquette (R.E.); Institut Universitaire de Cardiologie et de Pneumologie de Québec, Quebec, QC, Canada (F.P.); the Newcastle upon Tyne Hospitals, Newcastle upon Tyne (J.M.M.), and Liverpool Heart and Chest Hospital, Liverpool (D.J.W.) - both in the United Kingdom; Iowa Heart Center, West Des Moines (D. Sorrentino); Hartford Hospital, Hartford, CT (E.C.); Chula Vista Cardiac Center, Chula Vista, CA (B.B.); Arizona Arrhythmia Consultants, Scottsdale (D.R.); Policlinico Sant' Orsola-Malpighi, Bologna, Italy (M.B.); Baylor Research Institute, Plano, TX (H.K.); Oklahoma Heart Hospital, Oklahoma City (J.C.); MedStar Heart and Vascular Institute, Washington, DC (Z.E.); and Medtronic, Mounds View, MN (J.D.L., D.R.L., A.C.).

\section{REFERENCES}

1. Mond HG, Proclemer A. The 11th world survey of cardiac pacing and implantable cardioverter-defibrillators: calendar year 2009 - a World Society of Arrhythmia's project. Pacing Clin Electrophysiol 2011;34:1013-27.

2. Baddour LM, Epstein AE, Erickson CC, et al. Update on cardiovascular implantable electronic device infections and their management: a scientific statement from the American Heart Association. Circulation 2010;121:458-77.

3. Habib G, Lancellotti P, Antunes MJ, et al. 2015 ESC guidelines for the management of infective endocarditis: the Task Force for the Management of Infective Endocarditis of the European Society of Cardiology (ESC). Eur Heart J 2015;36: 3075-128.

4. Baman TS, Gupta SK, Valle JA, Yamada
E. Risk factors for mortality in patients with cardiac device-related infection. Circ Arrhythm Electrophysiol 2009;2:129-34. 5. Deckx S, Marynissen T, Rega F, et al. Predictors of 30-day and 1-year mortality after transvenous lead extraction: a singlecentre experience. Europace 2014;16:121825.

6. Tarakji KG, Wazni OM, Harb S, Hsu A, Saliba W, Wilkoff BL. Risk factors for 
1-year mortality among patients with cardiac implantable electronic device infection undergoing transvenous lead extraction: the impact of the infection type and the presence of vegetation on survival. Europace 2014;16:1490-5.

7. Sohail MR, Henrikson CA, Jo BraidForbes M, Forbes KF, Lerner DJ. Increased long-term mortality in patients with cardiovascular implantable electronic device infections. Pacing Clin Electrophysiol 2015; 38:231-9.

8. Kay G, Eby EL, Brown B, et al. Costeffectiveness of TYRX absorbable antibacterial envelope for prevention of cardiovascular implantable electronic device infection. J Med Econ 2018;21:294-300.

9. Bloom HL, Constantin L, Dan D, et al. Implantation success and infection in cardiovascular implantable electronic device procedures utilizing an antibacterial envelope. Pacing Clin Electrophysiol 2011; 34:133-42.

10. Henrikson CA, Sohail MR, Acosta $\mathrm{H}$ et al. Antibacterial envelope is associated with low infection rates after implantable cardioverter-defibrillator and cardiac resynchronization therapy device replacement: results of the Citadel and Centurion studies. JACC Clin Electrophysiol 2017;3: 1158-67.

11. Kolek MJ, Dresen WF, Wells QS, Ellis CR. Use of an antibacterial envelope is associated with reduced cardiac implantable electronic device infections in high-risk patients. Pacing Clin Electrophysiol 2013; 36:354-61.

12. Kolek MJ, Patel NJ, Clair WK, et al. Efficacy of a bio-absorbable antibacterial envelope to prevent cardiac implantable electronic device infections in high-risk subjects. J Cardiovasc Electrophysiol 2015; 26:1111-6.

13. Mittal S, Shaw RE, Michel K, et al. Cardiac implantable electronic device infections: incidence, risk factors, and the effect of the AigisRx antibacterial envelope. Heart Rhythm 2014;11:595-601. 14. Shariff N, Eby E, Adelstein E, et al. Health and economic outcomes associated with use of an antimicrobial envelope as a standard of care for cardiac implantable electronic device implantation. J Cardiovasc Electrophysiol 2015;26:783-9.

15. Tarakji KG, Mittal S, Kennergren C, et al. Worldwide Randomized Antibiotic EnveloPe Infection PrevenTion Trial (WRAP-IT). Am Heart J 2016;180:12-21.

16. Polyzos KA, Konstantelias AA, Falagas ME. Risk factors for cardiac implantable electronic device infection: a systematic review and meta-analysis. Europace 2015;17:767-77.

17. Head SJ, Kaul S, Bogers AJ, Kappetein AP. Non-inferiority study design: lessons to be learned from cardiovascular trials. Eur Heart J 2012;33:1318-24.

18. Kusumoto FM, Schoenfeld MH, Wilkoff
BL, et al. 2017 HRS expert consensus statement on cardiovascular implantable electronic device lead management and extraction. Heart Rhythm 2017;14(12): e503-e551.

19. Brunner MP, Cronin EM, Wazni O, et al. Outcomes of patients requiring emergent surgical or endovascular intervention for catastrophic complications during transvenous lead extraction. Heart Rhythm 2014;11:419-25.

20. Wazni O, Epstein LM, Carrillo RG, et al. Lead extraction in the contemporary setting: the LExICon study: an observational retrospective study of consecutive laser lead extractions. J Am Coll Cardiol 2010;55:579-86.

21. de Oliveira JC, Martinelli M, Nishioka $\mathrm{SA}$, et al. Efficacy of antibiotic prophylaxis before the implantation of pacemakers and cardioverter-defibrillators: results of a large, prospective, randomized, double-blinded, placebo-controlled trial. Circ Arrhythm Electrophysiol 2009;2:29-34.

22. Krahn AD, Longtin Y, Philippon F, et al. Prevention of Arrhythmia Device Infection Trial: the PADIT Trial. J Am Coll Cardiol 2018;72:3098-109.

23. Romeyer-Bouchard C, Da Costa A, Dauphinot V, et al. Prevalence and risk factors related to infections of cardiac resynchronization therapy devices. Eur Heart J 2010;31:203-10.

Copyright (@) 2019 Massachusetts Medical Society. 